Research Paper

\title{
Oxytocin inhibits ovarian cancer metastasis by repressing the expression of MMP-2 and VEGF
}

\author{
Haoyi $\mathrm{Ji}^{1}$, Na Liu ${ }^{2}$, Yingchun Yin ${ }^{3}$, Xinmei Wang ${ }^{3}$, Xiaoyang Chen ${ }^{1}$, Jing $\mathrm{Li}^{3 凶}$, Jingxin $\mathrm{Li}^{4}{ }^{\bowtie}$ \\ 1. Department of Medical Ethics, School of Medicine, Shandong University, Jinan 250012, China \\ 2. Department of Hematology, Qilu Hospital, Shandong University, Jinan 250012, China. \\ 3. Department of Pathology, Central Hospital of Zibo, Zibo 255036, China \\ 4. Department of Physiology, School of Medicine, Shandong University, Jinan 250012, China \\ $\square$ Corresponding authors: Jing Li, Department of Pathology, Central Hospital of Zibo, Zibo 255036, China. Tel: 86-533-2360323; Email: 1j13864152125@126.com; \\ Jingxin Li, Department of Physiology, School of Medicine, Shandong University, Jinan 250012, China. Tel: 86-531-88382395; Email: ljingxin@sdu.edu.cn \\ (1) Ivyspring International Publisher. This is an open access article distributed under the terms of the Creative Commons Attribution (CC BY-NC) license \\ (https://creativecommons.org/licenses/by-nc/4.0/). See http://ivyspring.com/terms for full terms and conditions.
}

Received: 2017.11.09; Accepted: 2018.02.04; Published: 2018.04.06

\begin{abstract}
Breastfeeding is associated with a decreased risk of ovarian cancer. However, the mechanism underlying this apparent clinical benefit is unknown. Oxytocin (OXT), a hypothalamic nonapetide, plays a crucial role in many reproductive and behavioural functions. In recent year, OXT acts as a growth regulator in many kind of tumor tissues, through the activation of a specific G-coupled transmembrane receptor, the oxytocin receptor (OXTR). OXT has been proved to inhibit the proliferation, migration and invasion of ovarian cancer SKOV3 cells in vitro. But, the underlying mechanisms remain unknown. Here, we found OXT inhibited proliferation, and critically migration and invasion of human ovarian cancer cells SKOV3 and A2780. Strikingly, OXT inhibited ovarian cancer metastasis by repressing the expressions of MMP-2 and VEGF. Moreover, OXT inhibited vascular endothelial cell tube formation by reducing the VEGF production from ovarian cancer cells. Our findings may provide a possible explanation for breastfeeding-associated protective effects and suggest new therapeutic opportunities for ovarian cancer.
\end{abstract}

Key words: Oxytocin; ovarian cancer; metastasis; MMP-2; VEGF

\section{Introduction}

Oxytocin (OXT) is the first peptide hormone structurally assessed and chemically synthesized in biologically active form. This hormone acts as an important factor in human reproductive system particularly during pregnancy and lactation in women. OXT is released during physical activity [1, 2], breastfeeding [3], pregnancy, etc. A review by Turck D [4] concluded that breastfeeding is associated with a decreased risk of breast and ovarian cancer in the premenopausal period. A case-control study in southern China revealed that prolonged lactation reduced ovarian cancer risk [5]. Harvard Center for Cancer Prevention also determined that women who breastfeed for more than one year accompanied with a decrease in ovarian cancer risk compared with those who never breastfeed [6]. Notably, a New England study with 563 participants also demonstrated that ever breastfeeding caused a reduction of ovarian cancer risk [7]. These clinical data strongly suggest that OXT is involved in breastfeeding-associated protect against ovarian cancer. Previous study has demonstrated that the oxytocin receptors (OXTRs) are expressed in human ovarian cancer cells [8]. Therefore, we speculate that the activation of OXTRs inhibits the progression of ovarian cancer. Although OXT inhibited the proliferation, migration and invasion of ovarian cancer as reported [8, 9], its underlying mechanisms had not fully elucidated. Morita et al. found that OXT inhibited the proliferation, migration and invasion of ovarian cancer by enhancing the expression of E-cadherin and slightly inhibiting MMP-2 in cultured SKOV3 cells [8]. Mankarious et al. revealed that OXT induced apoptosis in cultured three ovarian cancer cell lines 
including SKOV3, MDAH-2774 and PEO1[9]. Here we first reported that OXT suppressed the ovarian cancer metastasis by repressing not only the expression of MMP-2, but also angiogenesis in cultured A2780 cells. This finding may explain the ovarian cancerprotective effects of pregnancy or breastfeeding.

\section{Materials and Methods}

\section{Cell culture and reagents}

The human ovarian cancer cells SKOV3 and A2780 were donated by Dr. Zhaojian Liu from Department of Cell Biology, Shandong University School of Medicine, Jinan 250012, China. The SKOV3 cells were cultured in DMEM (Hyclone Co., Logan, UT, USA) supplemented with $10 \%$ (v/v) FBS (Gibco), $100 \mathrm{U} / \mathrm{ml}$ penicillin and $100 \mu \mathrm{g} / \mathrm{ml}$ streptomycin. The A2780 cells were cultured in RPMI 1640 (Hyclone Co.) supplemented with $10 \% \mathrm{FBS}, 100 \mathrm{U} / \mathrm{ml}$ penicillin and $100 \mu \mathrm{g} / \mathrm{ml}$ streptomycin. All cell lines were propagated at $37^{\circ} \mathrm{C}$ in an atmosphere containing $5 \%$ $\mathrm{CO}_{2}$. Primary antibodies for MMP2 and VEGF were purchased from Abcam (Austin, TX, USA). The $\beta$-actin and OXT were purchased from Sigma-Aldrich (St Louis, MO).

\section{Cell proliferation studies}

The proliferation of SKOV3 cells was assessed by Cell Counting Kit-8 (CCK-8, Beyotime, C0038) assay according to the protocol recommended by the manufacturer. This method is an optical density colorimetric assay that quantifies the number of viable cells based on the activity of cellular dehydrogenases. To estimate the number of viable cells, approximately 2000 cells were seeded in each well of 96-well plates with $100 \mu \mathrm{L}$ medium. OXT was added to each well with final concentrations of $0,10^{-7}, 10^{-6} \mathrm{M}$ for 48 hours. After treatment, $10 \mu \mathrm{L}$ CCK-8 solution was added into each well. Cells were then incubated for another 4 hours at $37^{\circ} \mathrm{C}$. The absorbance was measured by a microplate reader at the wave length of $450 \mathrm{~nm}$. In each experiment, six parallel wells were set up.

\section{Wound-healing assay}

Either SKOV3 or A2780 cells were seeded onto a six-well culture plate and cultured to a subconfluent state in complete medium. Cell monolayers were linearly scraped with a P-200 pipette tip $(250-\mu \mathrm{m}$ width). Cells detached from the bottom of the well were mildly aspirated and incubated in serum-free medium containing different concentrations of OXT $\left(0,10^{-9}, 10^{-8}, 10^{-7}\right.$ and $\left.10^{-6} \mathrm{M}\right)$ for 48 hours or for $0,6,12$, 18 and 24 hours with and without OXT $10^{-6} \mathrm{M}$ separately. The width of scratch was microscopically monitored at the various time points and quantified in terms of the difference between the original width of the wound and the width after cell migration. The percentage of wound closure [(original width-width after cell migration)/original width] was calculated. The width of the wound was measured using Image-Pro Plus 6.0.

\section{Transwell migration assay}

A complementary transwell migration assay was performed by employing a modified Boyden chamber (Corning Costar) containing a gelatin-coated polycarbonate membrane filter (pore size, $8 \mu \mathrm{m}$ ). A total of $2 \times 104$ cells in $500 \mu \mathrm{L}$ of culture medium containing various concentrations of OXT $\left(0,10^{-9}, 10^{-8}\right.$, $10^{-7}, 10^{-6} \mathrm{M}$ ) were added to the upper chamber, and the lower chamber contained culture medium with $10 \%$ FBS to stimulate cell migration. The migration assays were incubated for 24 hours at $37^{\circ} \mathrm{C}$ in $5 \% \mathrm{CO}_{2}$, and then the cells were stained with crystal violet. Cells on the undersides of the filters were observed under a microscope at a magnification of $200 \times$ and counted.

\section{Western blot}

For Western blot analysis, cells were lysed in lysis buffer [50 mM Tris ( $\mathrm{pH} 7.5), 100 \mathrm{mmol} / 1 \mathrm{NaCl}, 1$ mmol/1 EDTA, 0.5\% NP40, 0.5\% Triton X-100, 2.5 $\mathrm{mmol} / \mathrm{l}$ sodium orthovanadate, $10 \mu \mathrm{l} / \mathrm{ml}$ protease inhibitor cocktail, $1 \mathrm{mmol} / 1$ phenylmethylsulfonyl fluoride] for $30 \mathrm{~min}$ at $4^{\circ} \mathrm{C}$. Total proteins were fractionated by SDS-PAGE and transferred onto nitrocellulose membrane. The membranes were then incubated with appropriate primary antibodies (MMP2 or VEGF), followed by incubation with secondary HRP-conjugated antibody.

\section{Gelatin zymography analysis}

The effects of OXT on the gelatinolytic activities of MMP-2 were examined by gelatin zymography. A2780 cells were treated with various concentrations of OXT for 48 hours in serum-free medium. Serum-free supernatants were then harvested and centrifuged to remove cellular debris. Protein concentrations were determined with the bicinchoninic acid assay protein reagent kit (Sangon, Shanghai, China). An equal amount of protein $(20 \mu \mathrm{g})$ from each treatment was diluted with the loading buffer and fractionated on $10 \%$ SDS/PAGE containing $1 \mathrm{mg} / \mathrm{mL}$ gelatin A (Sigma Chemical Co.). After electrophoresis, the gels were stained with $0.1 \%$ Coomassie Brilliant Blue and destained with $45 \%$ methanol, $10 \%(\mathrm{v} / \mathrm{v})$ acetic acid until clear bands suggestive of gelatin digestion were present. 


\section{Matrigel in vitro HUVEC tube formation assay}

In vitro tube formation assay was performed according to the manufacturer's recommendation. Briefly, growth factor-reduced Matrigel, after being thawed on ice, was plated in pre-cooled 12-well chamber. After solidification of Matrigel (30 min incubation at $\left.37^{\circ} \mathrm{C}\right)$, HUVECs were trypsinized and seeded ( $5 \times 10^{4}$ cells per well) in each well with 1640 medium alone (control sample) or 1640 supplemented with cell-free culture supernatants from different treatment $(1: 1, \mathrm{v} / \mathrm{v})$. Each sample was added in duplicate wells. The chambers were then incubated at $37^{\circ} \mathrm{C}$ for 24 hours. After incubation and fixation phases, the tube formation process was scored using an inverted phase-contrast microscope and measured using Image-Pro Plus 6.0.

\section{Statistical analysis}

Continuous variables were recorded as mean \pm SD and analyzed by Student's $t$-test. Statistical analysis of the results was performed using GraphPad

A

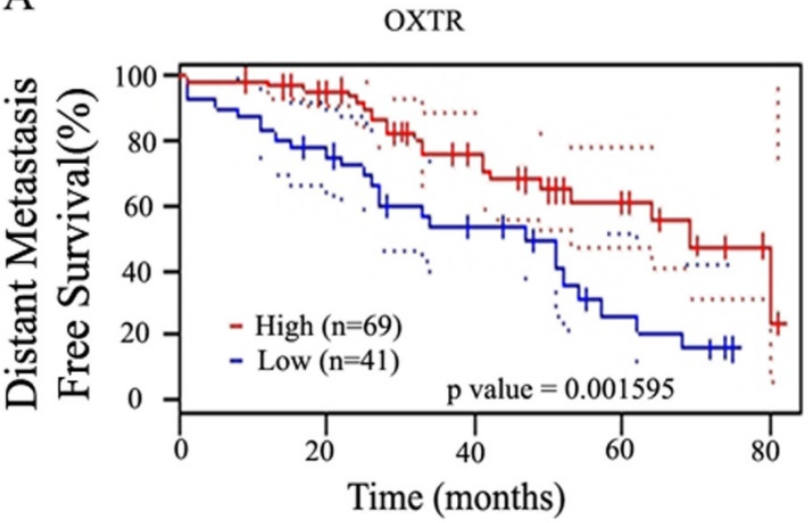

$\mathrm{C}$ SKOV3

$6 \mathrm{~h}$
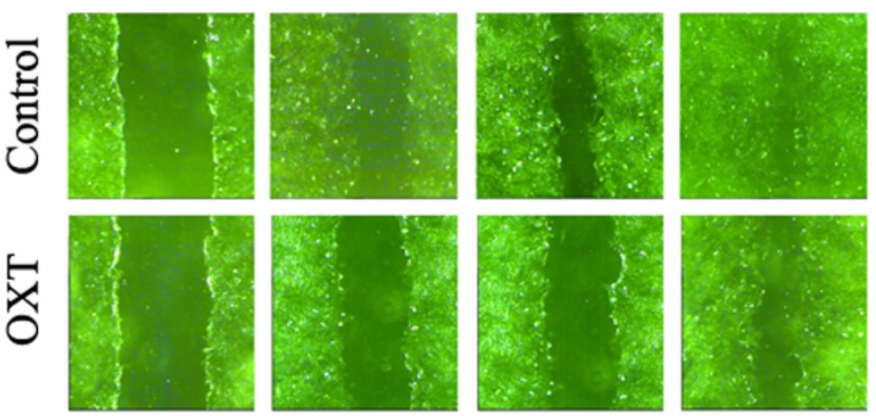

Fig. 1. OXT inhibited the proliferation and migration of human ovarian cancer SKOV3 cells. (A) Kaplan-Meier curves showing distant metastasis-free survival according to OXTR mRNA expression in ovarian cancer patients using in public gene dataset GSE17260[10]. Black, low expression; red, high expression (n represents the number of patients included in the analysis). (B) OXT dose-dependently inhibited the proliferation of human ovarian cancer SKOV3 cells, which was blocked by atosiban. (C) The effect of OXT on SKOV3 cells migration ability was plotted as the percentage of wound closure. The lines depict the mean \pm SD of three independent experiments. $*, n=5, P<0.05$. The result showed $O X T$ slowed down the scratch-induced wound healing.
StatMate software (GraphPad Software, Inc, San Diego, CA). The two-sided $P$-values $<0.05$ was considered statistically significant.

\section{Results}

\section{OXT inhibited the proliferation and invasion of ovarian cancer cells A2780 in vitro}

Kaplan-Meier curves show that the high level of OXTR in human ovarian tumors is associated with reduced metastasis-free survival (Fig.1A, based on public gene dataset GSE17260[10]), suggesting the activation of OXTR improves the outcome of human ovarian cancer. Indeed, we found that OXT inhibited human ovarian cancer SKOV3 cells proliferation in dose-response manner, which was blocked by atosiban, an OXTR antagonist (Fig. 1B, n=5). OXT also delayed the scratch-induced wound healing in SKOV3 cells (Fig.1C, $n=5$ ). Moreover, OXT also markedly suppressed the migration and invasion of human ovarian cancer cell line A2780 (Fig. 2A-B).

B
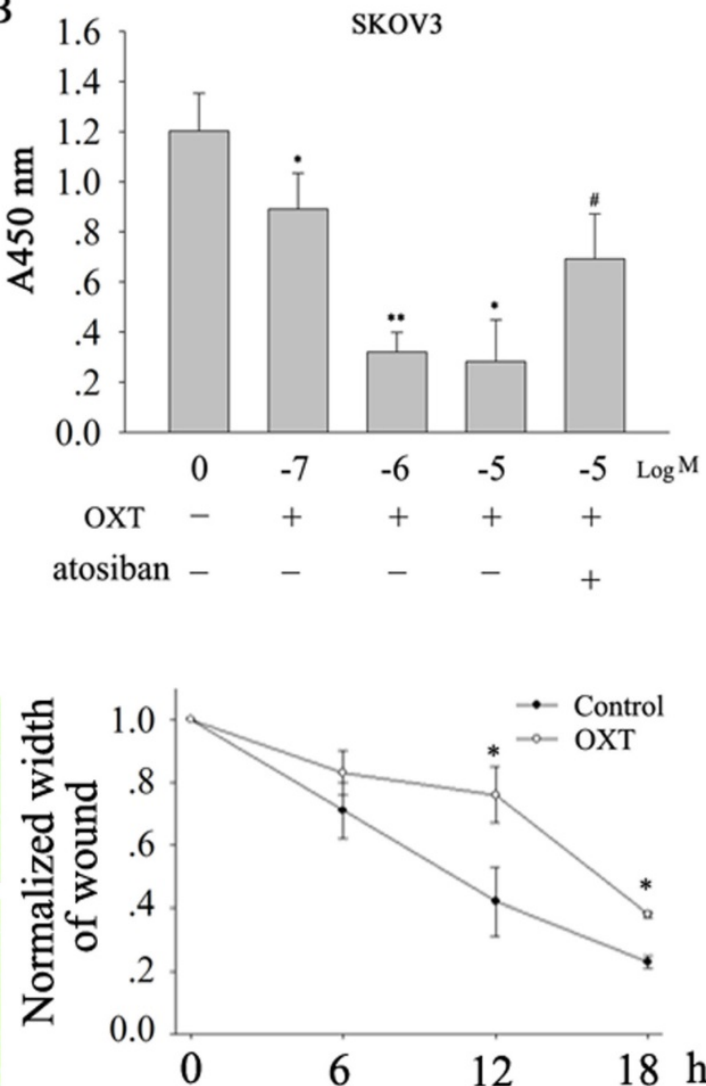


\section{OXT decreased the production of MMP-2 in A2780}

Utilizing Western blotting analysis and the SDS-PAGE gelatin zymography, we revealed that OXT dose-dependently inhibited the MMP-2 protein expression and enzyme activity in human ovarian cancer cells of A2780 (Fig. 3A-B).
OXT inhibited the VEGF production in human ovarian cancer cells of A2780 and human endothelial cell tube formation

OXT also dose-dependently inhibited the expression of VEGF (Fig. 4A) in human ovarian cancer cells of A2780. Strikingly, OXT slowed the human endothelial cell (HUVEC) tube formation induced by conditioned medium collected from A2780 (Fig. 4B).
A

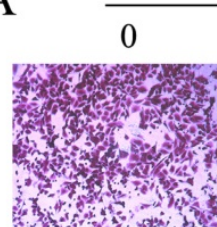

B
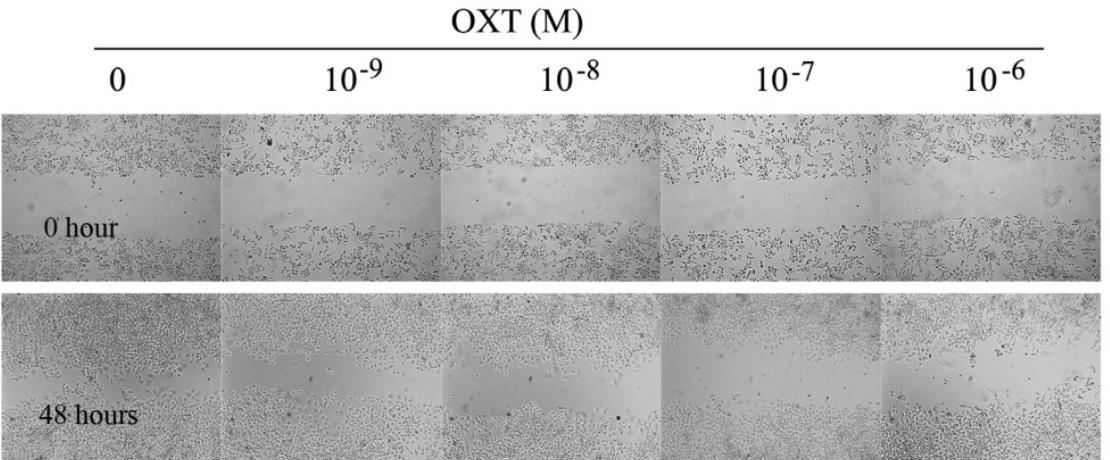

OXT (M)

$10^{-8}$

$10^{-7}$

$10^{-6}$
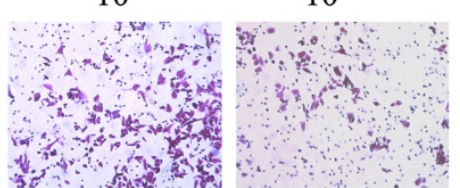

$\operatorname{OXT}(\mathrm{M})$

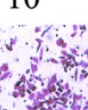

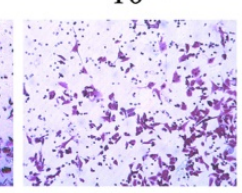

$10^{-7}$
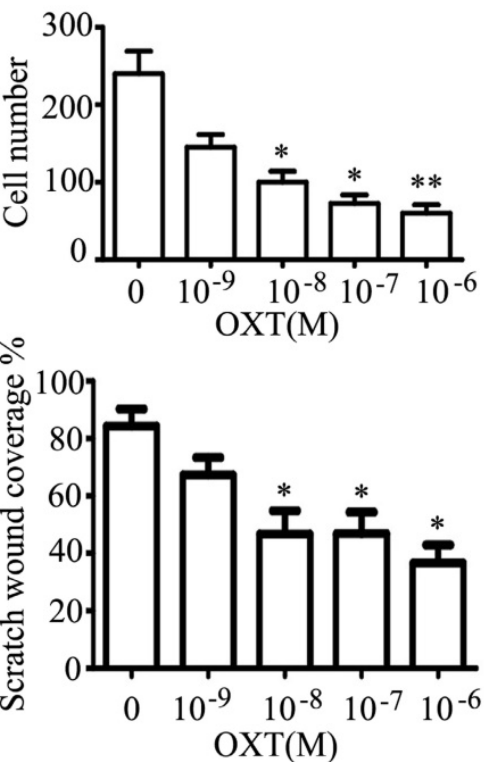

Fig. 2. OXT suppressed the invasion and migration of human ovarian cancer cell line A2780. (A) A2780 cells were seeded in the upper chamber treated with $0,10^{-9}, 10^{-8}, 10^{-7}$ and 10-6 $\mathrm{M}$ of OXT and allowed to migrate for 24 hours; then, the migrated cells were stained with crystal violet. OXT dose-dependently depressed the invasion of human ovarian cancer cell line A2780. The graphs depict the mean \pm SD of three independent experiments. *, $P<0.05$. (B) The effect of oxytocin on A2780 cells cells migration ability was plotted as the percentage of wound closure. OXT reduced the scratch-induced wound healing in human ovarian cancer cell line A2780. The graphs depict the mean \pm SD of three independent experiments. *, $P<0.05$.

A Western blot analysis
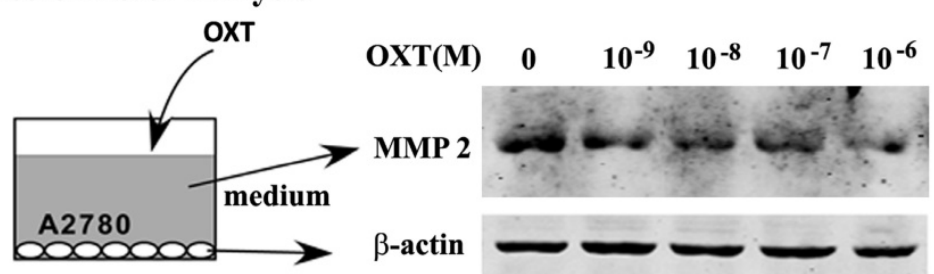

64KD

43KD

B Gelatin zymography analysis

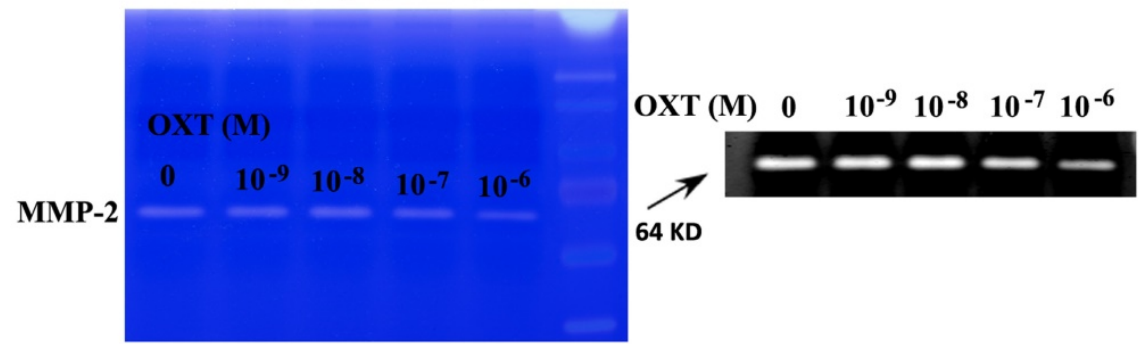

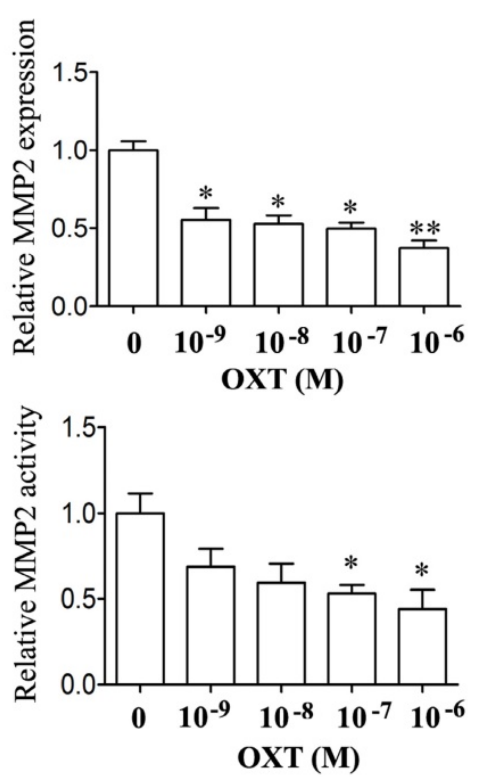

Fig. 3. OXT inhibited MMP-2 production. (A) A2780 cells were treated for 0, 10-9, 10-8, 10-7 and 10-6 $M$ of OXT for 48 hours. The cells were lysed and processed on a Western blot and probed for MMP-2. (B) A2780 cells were treated for $0,10^{-9}, 10^{-8}$, 10-7 and 10-6 M of OXT for 48 hours. MMP-2 activities in OXT-treated cells were detected using gelatin zymography analysis. Mean densitometry from 3 independent experiments demonstrated that OXT decreased MMP-2 production $(* P<0.05)$ 
A Western blot analysis
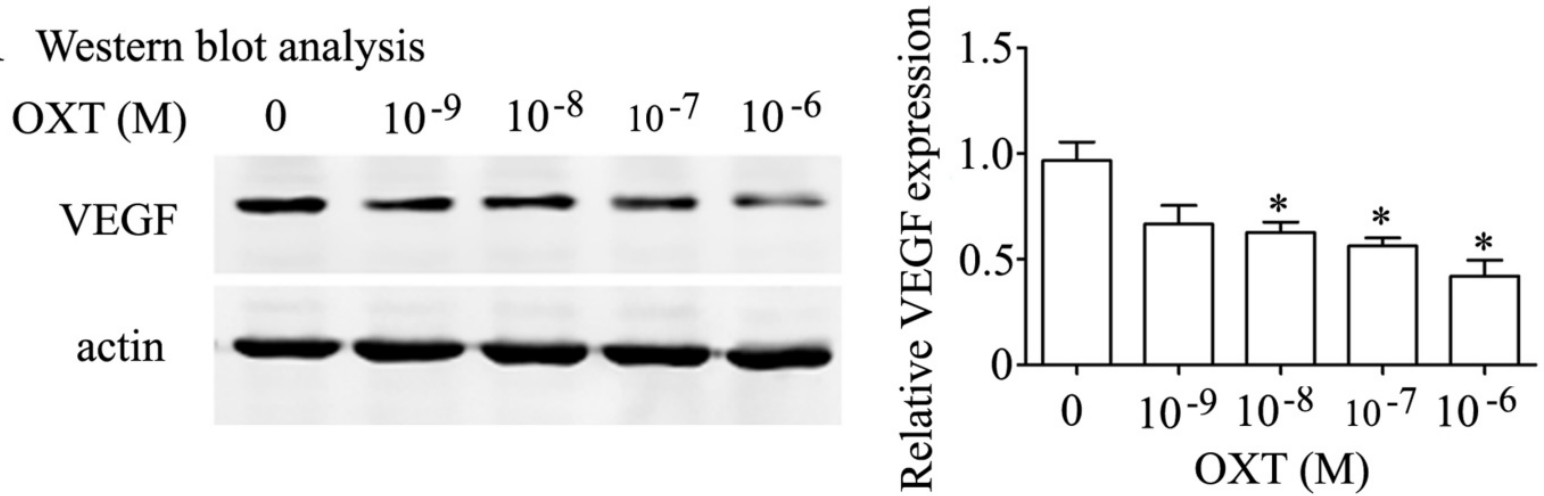

B Tube formation assay
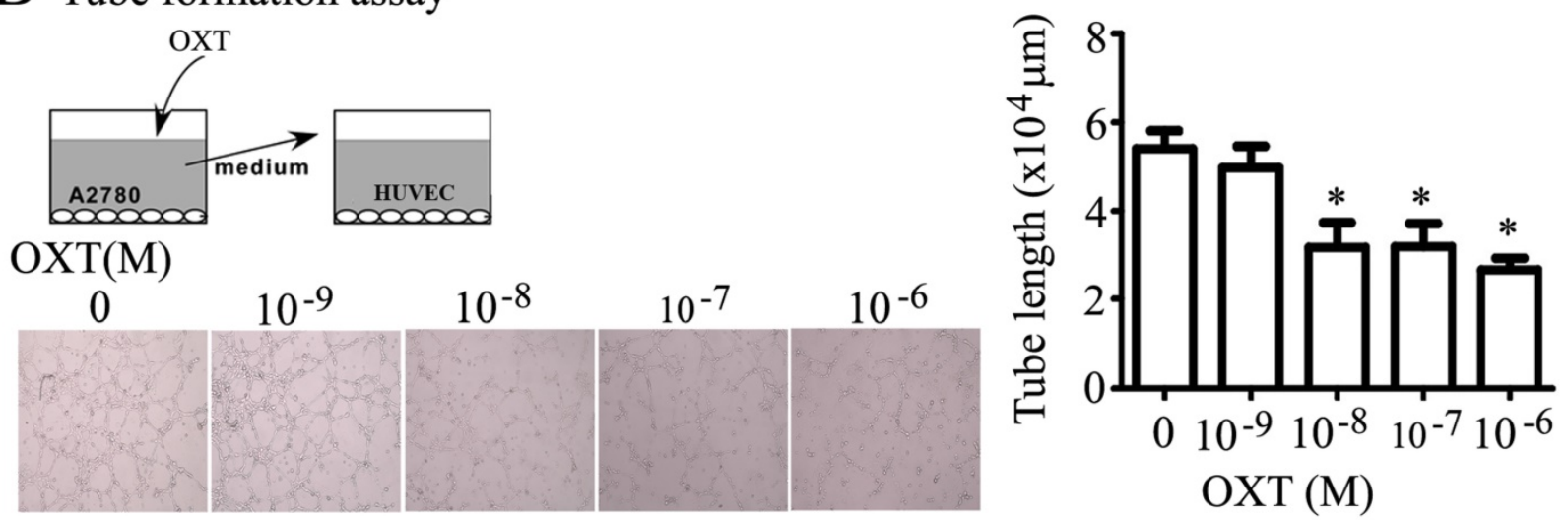

Fig. 4. Effect of OXT on VEGF and angiogenesis activity in vitro. (A) A2780 cells were treated for $0,10^{-9}, 10^{-8}, 10^{-7}$ and $10^{-6} \mathrm{M}$ of OXT for 48 hours. The cells were lysed and processed on a Western blot and probed for VEGF.(B) Angiogenesis was measured by in vitro tube formation assay. HUVECs were cultured with supernatants of A2780 cells treated with serial concentration OXT on Matrigel. The degree of tube formation was quantified by Image-Pro Plus 6.0. The graphs depict the mean \pm SD of three independent experiments. *, $P<0.05$.

\section{Discussion}

Although several decades of clinical experience strongly suggest that parity and breastfeeding are beneficial for ovarian cancer patients [11-13], the mechanisms through which pregnancy, or lactation, influences ovarian cancer have gone relatively unexplored. So far, we are unaware of any previous efforts to harness the ovarian cancer-protective effects of pregnancy or breastfeeding for therapeutic benefit. Here we first demonstrated that the high level of OXTR in human ovarian tumors is associated with reduced metastasis-free survival, hinting that OXT signal pathway may involve in metastasis of human ovarian tumors. Next, we revealed that OXT inhibited the proliferation, migration and invasion of human ovarian cancer cells SKOV3 and A2780, which is consistent with finding of Morita et al. [8] in the ovarian cancer cell line SKOV3. Importantly, in our present study, we determined that one of the major mechanisms through which OXT antagonizes ovarian cancer is through VEGF pathway. OXT significantly reduced the VEGF production from ovarian cancer cells. VEGF is a key angiogenic growth factor that stimulates proliferation, migration, and capillary tube formation [14]. As expected, OXT indeed inhibited angiogenesis in vitro. Our finding may provide a plausible explanation for the ovarian cancerprotective effects of pregnancy or breastfeeding.

Morita et al. demonstrated that OXT inhibited the expression of MMP-2 in human ovarian cancer cells SKOV3 [8]. Notably, utilizing Western blotting analysis and the SDS-PAGE gelatin zymography we had got the similar results in another human ovarian cancer cell line A2780. In the initial step of tumor cell invasion, the release of proteolytic enzymes is required for the detachment from the extracellular matrix [15]. Thereby, the OXT-induced inhibition of ovarian cancer migration is possibly via the decrease of matrix synthesis. To our knowledge, this is the first work to demonstrate the role of OXT on the VEGF production of ovarian cancer cells and angiogenesis, and this may provide a possible explanation for breastfeeding-associated protective effects and prove useful for ovarian cancer treatment.

\section{Acknowledgements}

This research was supported by the National Natural Science Foundation of China (NSFC31600940) and Natural Science Foundation of Shandong Province (No. ZR2014HP069). 


\section{Competing Interests}

The authors have declared that no competing interest exists.

\section{References}

1. Hew-Butler T, Noakes TD, Soldin SJ, Verbalis JG. Acute changes in endocrine and fluid balance markers during high-intensity, steady-state, and prolonged endurance running: unexpected increases in oxytocin and brain natriuretic peptide during exercise. European journal of endocrinology. 2008;159:729-37.

2. Gutkowska J, Paquette A, Wang D, Lavoie JM, Jankowski M. Effect of exercise training on cardiac oxytocin and natriuretic peptide systems in ovariectomized rats. American journal of physiology Regulatory, integrative and comparative physiology. 2007;293:R267-75.

3. Jong TR, Menon R, Bludau A, Grund T, Biermeier V, Klampfl SM, et al. Salivary oxytocin concentrations in response to running, sexual self-stimulation, breastfeeding and the TSST: The Regensburg Oxytocin Challenge (ROC) study. Psychoneuroendocrinology. 2015;62:381-8.

4. Turck D, Comite de nutrition de la Societe francaise de p. [Breast feeding: health benefits for child and mother]. Archives de pediatrie : organe officiel de la Societe francaise de pediatrie. 2005;12 Suppl 3:S145-65.

5. Su D, Pasalich M, Lee AH, Binns CW. Ovarian cancer risk is reduced by prolonged lactation: a case-control study in southern China. The American journal of clinical nutrition. 2013;97:354-9.

6. Colditz GA, Atwood KA, Emmons K, Monson RR, Willett WC, Trichopoulos D, et al. Harvard report on cancer prevention volume 4: Harvard Cancer Risk Index. Risk Index Working Group, Harvard Center for Cancer Prevention. Cancer causes \& control : CCC. 2000;11:477-88

7. Titus-Ernstoff L, Perez K, Cramer DW, Harlow BL, Baron JA, Greenberg ER. Menstrual and reproductive factors in relation to ovarian cancer risk. British journal of cancer. 2001;84:714-21.

8. Morita T, Shibata K, Kikkawa F, Kajiyama H, Ino K, Mizutani S. Oxytocin inhibits the progression of human ovarian carcinoma cells in vitro and in vivo. International journal of cancer. 2004:109:525-32.

9. Mankarious A, Dave F, Pados G, Tsolakidis D, Gidron Y, Pang Y, et al. The pro-social neurohormone oxytocin reverses the actions of the stress hormone cortisol in human ovarian carcinoma cells in vitro. International journal of oncology. 2016;48:1805-14.

10. Mizuno H, Kitada K, Nakai K, Sarai A. PrognoScan: a new database for meta-analysis of the prognostic value of genes. BMC medical genomics. 2009;2:18.

11. Kim SJ, Rosen B, Fan I, Ivanova A, McLaughlin JR, Risch H, et al. Epidemiologic factors that predict long-term survival following a diagnosis of epithelial ovarian cancer. British journal of cancer. 2017;116:964-71.

12. Koushik A, Grundy A, Abrahamowicz M, Arseneau J, Gilbert L, Gotlieb WH, et al. Hormonal and reproductive factors and the risk of ovarian cancer. Cancer causes \& control : CCC. 2017;28:393-403.

13. Sung HK, Ma SH, Choi JY, Hwang Y, Ahn C, Kim BG, et al. The Effect of Breastfeeding Duration and Parity on the Risk of Epithelial Ovarian Cancer: A Systematic Review and Meta-analysis. Journal of preventive medicine and public health = Yebang Uihakhoe chi. 2016;49:349-66.

14. Ushio-Fukai M. Redox signaling in angiogenesis: role of NADPH oxidase. Cardiovascular research. 2006;71:226-35

15. Yano $T$, Yamasaki $H$. Regulation of cellular invasion and matrix metalloproteinase activity in HepG2 cell by connexin 26 transfection. Molecular carcinogenesis. 2001;31:101-9. 